Itinéraires Itinéraires

Littérature, textes, cultures

\title{
Richard Bauman et Charles L. Briggs, Voices of Modernity. Language Idéologies and the Politics of Inequality
}

Xavier Garnier

\section{OpenEdition}

Journals

Édition électronique

URL : http://journals.openedition.org/itineraires/562

DOI : 10.4000/itineraires.562

ISSN : 2427-920X

Éditeur

Pléiade

\section{Édition imprimée}

Date de publication : 1 novembre 2009

Pagination : 185-187

ISBN : 978-2-296-10115-9

ISSN : 2100-1340

Référence électronique

Xavier Garnier, «Richard Bauman et Charles L. Briggs, Voices of Modernity. Language Idéologies and the Politics of Inequality », Itinéraires [En ligne], 2009-3 | 2009, mis en ligne le 26 juin 2014, consulté le 22 septembre 2020. URL : http://journals.openedition.org/itineraires/562 ; DOI : https://doi.org/10.4000/ itineraires.562

\section{(2) $\odot \Theta \Theta$}

Itinéraires est mis à disposition selon les termes de la licence Creative Commons Attribution - Pas d'Utilisation Commerciale - Pas de Modification 4.0 International. 
Ces réminiscences de sa poétique proche de celle des Cultural Studies (fondée sur la prise en compte du cadre historique et interprétatif ${ }^{1}$ ), l'amènent à conclure sur un appel marxiste à la dynamique sociale de l'utopie en appelant les ontologies du présent à se tourner vers des archéologies du futur au lieu des prédictions $d u$ passé, annonçant par là son prochain ouvrage : Archeologies of the Future (traduit en français sous le titre Archéologies du Futur, Max Milo, 2007).

\section{Maëline Le Lay \\ Université Paris 13 - CENEL}

Richard Bauman et Charles L. Briggs, Voices of Modernity. Language Ideologies and the Politics of Inequality, Cambridge, Cambridge University Press, 2003, 356 p.

Parce qu'ils travaillent dans le champ de l'anthropologie culturelle, Bauman et Briggs sont bien placés pour être sensibles au paradoxe qui sous-tend la notion de «langage traditionnel»: sur le terrain, en enquêtant sur tel ou tel peuple, ils peuvent sentir la puissance performative de la parole dite traditionnelle et pour autant, leurs catégories méthodologiques et conceptuelles les contraignent à cantonner leur objet d'étude dans l'espace. Comme si, quel que soit le génie déployé par l'orateur, une force invisible empêchait sa parole de déborder ce cadre neutralisateur de la tradition.

L'ambition des auteurs de cet ouvrage est de comprendre selon quelles procédures s'est construite, à partir du XVII ${ }^{\mathrm{e}}$ siècle, l'idée d'une parole moderne à partir d'une différenciation d'avec l'ensemble multiple des paroles traditionnelles, prémodernes, folkloriques. Le travail sur ces paroles « archaïques » est une pièce maîtresse de la construction de la modernité. Bauman et Briggs veulent montrer comment la conception d'un langage moderne naît d'un travail de purification qui en fait un outil clé de la structuration des relations sociales et de la politique d'inégalité entre les groupes sociaux. L'identification de "l'Autre pré-moderne » à la fois à l'intérieur des sociétés modernes (ruraux, pauvres, illettrés...) et à l'extérieur (sauvages, primitifs...) est fondée sur une poétique de l'altérité mise au jour par la tradition orale. En ce sens les études folkloristes sont moins le fait de nostalgiques de mondes anciens qu'une pièce maîtresse dans la construction d'un espace social de la modernité.

Bauman et Briggs voient en Locke le véritable initiateur de cette politique d'inégalité linguistique : à la différence de Francis Bacon, qui voit dans le langage un obstacle à une connaissance scientifique et moderne des phénomènes naturels, le Locke de l'Essai sur l'entendement humain revendique pour le langage un espace neutre, une " province séparée » totalement décontextualisée du corps social, où les signes purifiés pourraient servir à construire un savoir moderne sur le monde.

1. Fredric Jameson, The Political Unconscious Narrative as a Socially Symbolic Act, Cornell University Press, 1981. 
Bauman et Briggs montrent comment «Locke's regime of decontextualization seemed to free some individuals from their social circumstances and from the chains of tradition and render them cosmopolitan subjects, able to speak to and for the world, for "man" » (p. 59).

Dans le troisième chapitre de l'ouvrage, Bauman et Briggs repartent de Francis Bacon et de la rupture qu'il pointe entre la science ancienne et la science moderne, pour distinguer deux types d'approches des mondes anciens à la charnière des XVII et $\mathrm{XVIII}^{\mathrm{e}}$ siècles : celle des antiquisants et celle des philologues. Les antiquisants (John Aubrey, Henry Bourne, John Brand) travaillent sur les restes du passé qui leur arrivent par fragments isolés et témoignent des logiques symboliques qui présidaient à des mondes disparus. Ces monuments hérités de mondes passés, témoins d'âges obscurs livrés à l'ignorance, peuvent rester actifs dans des sphères sociales intégrées au monde moderne, elles-mêmes par là même monumentalisées. Les philologues (Thomas Blackwell, Robert Wood, Robert Lowth) se distinguent des antiquisants par leur souci du texte et du jeu des formes, d'où dérive la mise en forme de ces mondes anciens comme « contextes ». La perspective philologique permet la mise en relations des mondes par le biais de la comparaison des formes et donne ainsi naissance à un relativisme culturel solidaire d'un positionnement analytique global. Le travail sur l'Orient effectué par la philologie moderne est un chantier crucial dans la constitution d'une perspective relativiste moderne sur le langage figuratif, par exemple sur les conditions d'appréciation du langage poétique.

La synthèse entre les points de vue antiquisants et philologiques est présentée dans le quatrième chapitre consacré au travail de Hughes Blair sur les poèmes d'Ossian publiés par Macpherson. Précisément parce que ces poèmes sont censés être des fragments isolés sauvés de la disparition du monde celte, ils sont chargés de la poéticité des origines et sont susceptibles de nourrir un renouveau national écossais :

The poems, songs, and tales, in the vernacular repertoire are highly valued as national symbols, but in their hybrid state - distressed remnants of the past in the present - they cannot serve this important function without the intervention, mediation, and authorization of intellectuals. (p. 162)

Recontextualiser un monde moderne autour de ces fragments du passé, telle va être la tâche de ces intellectuels. Le cinquième chapitre, consacré à la pensée de Johann Gottfried Herder, montre la dette du philosophe allemand à l'égard de la pensée philologique britannique notamment concernant sa conception de la tradition comme constitutive de la littérature vernaculaire et de l'identité nationale et du rôle des intellectuels dans la construction de cette tradition. Prenant acte de tout ce qui sépare Locke et Herder quant à la conception du langage et du savoir modernes (purs et décontextualisés chez le premier, à la fois ancrés dans la nature et la société chez le second), Bauman et Briggs insistent sur la convergence entre les deux philosophes quant à la nécessité d'une médiation métadiscursive pour construire un ordre supérieur de rationalité moderne. 
Le travail effectué par les frères Grimm au début du XIX ${ }^{\mathrm{e}}$ siècle révèle de façon éclairante comment les Européens parviennent à « déprovincialiser » (pour reprendre l'expression de Chakrabarty et Chatterjee) leurs propres cultures non en déclarant leur universalité, mais en affirmant leur provincialité intrinsèque. En asseyant leur autorité de philologues, en convertissant la mémoire orale en textes imprimés, en stabilisant un corpus idéalement à la fois authentique et exhaustif, en rendant disponible par la publication ce répertoire traditionnel, les frères Grimm participent à la constitution d'une nation moderne complexe, unifiée, organique, capable de se rassembler autour d'un corps textuel scientifiquement édité : la synthèse entre Locke et Herder est pratiquement effectuée. Cette mise sur le marché d'un corpus de textes établissant un lien avec le passé et consommables par un public bourgeois lettré est constitutive de la généalogie des États modernes.

La démarche des frères Grimm trouve un prolongement révélateur dans celle de Henry Rowe Schoolcraft (1793-1864) qui, à une époque où était assurée la suprématie des colons américains sur les peuples indiens, entreprit un considérable travail de collecte et d'édition des traditions orales amérindiennes. Ce travail qui tente de tenir ensemble l'authenticité scientifique et la lisibilité littéraire dans l'établissement de textes publiés a la double ambition de fonder une ethnographie américaine et d'influer sur le développement d'une indépendance littéraire nationale sur la scène poétique internationale, en mettant à la disposition des écrivains une source de formes littéraires inédites.

L'ouvrage s'achève sur un exposé des conceptions de Franz Boas pour une anthropologie cosmopolite affranchie de l'enfermement culturel apportée par la notion de tradition. Dans la perspective de Boas, en cela héritier direct de Locke, les cultures doivent être délivrées de l'emprise de la tradition par l'établissement de textes en langues vernaculaires, mettant à la disposition de la communauté scientifique le substrat phonétique commun à toutes les langues du monde et permettant une description et une comparaison purement analytique. Avec Boas, l'anthropologie ne se défait pas de l'idée de culture, mais dénoue le lien entre culture et tradition : mettre la culture en textes, c'est ouvrir la voie à une science cosmopolite des textes, qui doit faire de l'anthropologie une science irréductible à l'instrumentalisation nationaliste.

La recherche de Bauman et Briggs ne cache pas sa dimension politique. L'enquête qu'ils mènent sur l'évolution des idéologies du langage dans leur lien avec l'idée de modernité, a le grand mérite d'attirer notre attention sur la nature nécessairement asymétrique du partage entre modernité et prémodernité : pour construire sa transparence et sa pureté la parole moderne a besoin de montrer l'hybridité intrinsèque de la parole de l'Autre (intérieur ou extérieur), de la mettre en scène et d'en faire l'expertise. La modernité est solidaire de cet avènement de la parole prémoderne, qui lui sert tout autant de repoussoir que de fondement. 